\title{
REGULARIZATION OF NONLINEAR ILL-POSED PROBLEMS BY EXPONENTIAL INTEGRATORS
}

\author{
Marlis Hochbruck ${ }^{1}$, Michael Hönig ${ }^{1}$ And Alexander Ostermann ${ }^{2}$
}

\begin{abstract}
The numerical solution of ill-posed problems requires suitable regularization techniques. One possible option is to consider time integration methods to solve the Showalter differential equation numerically. The stopping time of the numerical integrator corresponds to the regularization parameter. A number of well-known regularization methods such as the Landweber iteration or the Levenberg-Marquardt method can be interpreted as variants of the Euler method for solving the Showalter differential equation.

Motivated by an analysis of the regularization properties of the exact solution of this equation presented by Tautenhahn [22], we consider a variant of the exponential Euler method for solving the Showalter ordinary differential equation. We discuss a suitable discrepancy principle for selecting the step sizes within the numerical method and we review the convergence properties of [22] and of our discrete version [8]. Finally, we present numerical experiments which show that this method can be efficiently implemented by using Krylov subspace methods to approximate the product of a matrix function with a vector.
\end{abstract}

1991 Mathematics Subject Classification. 65J20, 65N21, 65L05.

The dates will be set by the publisher.

\section{INTRODUCTION}

In this paper we consider the problem

$$
F(u)=y,
$$

where $F: \mathcal{D}(F) \subset X \rightarrow Y$ is a nonlinear operator between two Hilbert spaces $X$ and $Y$. We always require that the operator $F$ is Fréchet differentiable and possesses a locally uniformly bounded Fréchet derivative $F^{\prime}(u)$. We further assume that (1) has a solution in $\mathcal{D}(F)$ but we do not assume that this solution is unique. Our main interest is in ill-posed problems, where the solution does not depend continuously on the data $y$. In this case the solution of the perturbed problem

where the perturbed data $y^{\delta} \approx y$ satisfies

$$
F\left(u^{\delta}\right)=y^{\delta},
$$

$$
\left\|y^{\delta}-y\right\| \leq \delta
$$

Keywords and phrases: nonlinear ill-posed problems, asymptotic regularization, exponential integrators, variable step sizes, convergence, optimal convergence rates

1 Mathematisches Institut, Heinrich-Heine Universität Düsseldorf, Universitätsstraße 1, D-40225 Düsseldorf, Germany;

e-mail: marlis@am.uni-duesseldorf.de \& hoenig@am.uni-duesseldorf.de

2 Institut für Mathematik, Universität Innsbruck, Technikerstraße 13, A-6020 Innsbruck, Austria;

e-mail: alexander.ostermann@uibk.ac.at 
requires suitable regularization techniques. Throughout the paper, we denote the inner products in $X$ and $Y$ by $\langle\cdot, \cdot\rangle$ and the corresponding norms by $\|\cdot\|$, respectively. It is always clear from the context which norm is meant.

One of the most popular regularization methods is Tikhonov regularization, where an approximation $u_{\alpha}^{\delta}$ to the searched solution is defined as a solution of the minimization problem

$$
\min _{u^{\delta} \in \mathcal{D}(F)}\left(\left\|F\left(u^{\delta}\right)-y^{\delta}\right\|^{2}+\alpha\left\|u^{\delta}-u_{0}\right\|^{2}\right) .
$$

The real number $\alpha>0$ is a suitably chosen regularization parameter, the function $u_{0}$ is a given approximation to a solution of (1). For nonlinear problems, an analysis of Tikhonov regularization is presented in $[4,15,20]$. Other options for regularization are variants of inexact Newton methods [12,16,17]. A nice review is given in the recent book by Kaltenbacher et al. [14].

In this paper, we consider an asymptotic regularization based on the Showalter differential equation [21]

$$
\frac{\mathrm{d}}{\mathrm{d} t} u^{\delta}(t)=-F^{\prime}\left(u^{\delta}(t)\right)^{*}\left(F\left(u^{\delta}(t)\right)-y^{\delta}\right), \quad t \geq 0, \quad u^{\delta}(0)=u_{0}
$$

The regularization is performed by solving this differential equations up to a certain time $T$ only. This stopping time is used as regularization parameter. Solving the above initial value problem numerically with some time integration method like explicit or implicit Runge-Kutta methods leads to a variety of well-known regularization schemes. For instance, the Landweber iteration is equivalent to solving (4) by the forward Euler method [7]. Tikhonov regularization and the Levenberg-Marquardt scheme can be interpreted as the application of a linearly implicit Euler method [6]. We will discuss these relationships in more detail in Section 1 below.

Recently, the use of Rosenbrock methods has been studied in [1], and Runge-Kutta methods for linear problems have been analyzed in [19]. However, using explicit schemes has the major drawback that they suffer from severe step size restrictions to ensure stability. Fully implicit schemes, on the other hand, are difficult to analyze for nonlinear problems. A further difficulty arises when the time-integration scheme is used with variable step sizes. To the best of our knowledge, there is no result on optimal convergence rates for this situation.

Our motivation for this paper was twofold. First, it was shown in [22] that the exact solution of (4) yields an optimal order regularization scheme. Second, the problem considered here seems to be related to our recent work [11] on exponential integrators for nonlinear parabolic problems, since the operator $-F^{\prime}\left(u^{\delta}\right)^{*} F^{\prime}\left(u^{\delta}\right)$ approximating the Jacobian of the right-hand side of (4) has its spectrum on the negative real axis. However, there is one major difference: In the analysis of parabolic problems we are faced with large norms of the Jacobian and small step sizes whereas in the analysis of ill-posed problems, the norm of the Jacobian is small (we will later assume that $\left\|F^{\prime}(u)\right\| \leq 1$ ) but one is interested in using large time steps. Thus the convergence theory of [11] cannot be applied directly.

An outline of the paper is as follows: In Section 1, we review the role of time integration schemes as regularizing methods. In Section 2, we introduce the exponential Euler method as a new regularizing method for nonlinear ill-posed problems. We further show that the method can be interpreted as Newton's method with asymptotic regularization for the linearized problems. Our error analysis is partly based on the framework and results of Tautenhahn for the continuous problem. This work will be reviewed in Section 3. Our new convergence results are given in Section 4. We obtain convergence for a variable step size implementation of the exponential Euler method, and we present optimal convergence rates. The proofs of our convergence results, however, are quite involved and will appear elsewhere, see [8]. In Section 5 we give some hints on the implementation. In particular, the evaluation of certain matrix functions requires care. Section 6 is devoted to numerical experiments. We consider the identification of a coefficient in an elliptic PDE and an inverse problem from groundwater hydrology. 


\section{Time integration schemes as Regularization methods}

One possible approach to regularize the ill-posed problem (2) is asymptotic regularization or Showalter's method [5,21]. The idea behind this method is to solve the initial value problem (4) up to a certain time $t=T$. The stopping time $T$ serves as a regularization parameter and will be chosen such that the discrepancy principle is satisfied, i.e.

$$
\left\|F\left(u^{\delta}(T)\right)-y^{\delta}\right\| \leq \tau \delta<\left\|F\left(u^{\delta}(t)\right)-y^{\delta}\right\|, \quad 0 \leq t<T
$$

with some $\tau>2$, see, e.g., [4]. The aim of this paper is to explore the potential of exponential integrators for the numerical solution of the Showalter equation (4). To improve the readability of the paper, we denote in the following

$$
J(u)=F^{\prime}(u)^{*} F^{\prime}(u), \quad K(u)=F^{\prime}(u) F^{\prime}(u)^{*}
$$

Employing standard time-integration schemes for solving (4) is not a new idea. In fact, a number of wellknown regularization schemes can be interpreted as numerical methods for solving (4). The first (and simplest) example of such a scheme is the explicit Euler method given by

$$
u_{n+1}^{\delta}=u_{n}^{\delta}-h_{n} F^{\prime}\left(u_{n}^{\delta}\right)^{*}\left(F\left(u_{n}^{\delta}\right)-y^{\delta}\right), \quad t_{n+1}=t_{n}+h_{n}, \quad n=0,1,2, \ldots
$$

Here, $u_{n}^{\delta}$ is an approximation to the exact solution of (4) at time $t=t_{n}$. For constant step sizes $h_{n}=1$, this method is just the Landweber iteration. In [7], its convergence was analyzed, and it was proved that the scheme converges with optimal rates in terms of $\delta$.

A major drawback of the explicit Euler method is that, roughly speaking, stability can be guaranteed only for step sizes $h_{n}$ satisfying $h_{n}\left\|J\left(u_{n}^{\delta}\right)\right\| \leq 2$. This means that a large number of steps is required whenever $T$ is large. An alternative would be to use the following variant of the linearly implicit Euler scheme

$$
u_{n+1}^{\delta}=u_{n}^{\delta}-h_{n}\left(I+h_{n} J\left(u_{n}^{\delta}\right)\right)^{-1} F^{\prime}\left(u_{n}^{\delta}\right)^{*}\left(F\left(u_{n}^{\delta}\right)-y^{\delta}\right), \quad t_{n+1}=t_{n}+h_{n}, \quad n=0,1,2, \ldots
$$

We remark that $J\left(u_{n}^{\delta}\right)$ is only an approximation to the Jacobian of the right-hand side of (4). This scheme is equivalent to the Levenberg-Marquardt iteration. In [6] a convergence analysis is presented for variable step sizes $h_{n}$ chosen appropriately. Note that, if Assumption 1 below holds, then there is a unique solution $u_{+}$of (1) with minimal distance to $u_{0}$. We assume that for $\mu_{n}<1<\nu_{n}$, the error $e_{n}=u_{n}^{\delta}-u_{+}$after $n$ iterations satisfies

$$
\left\|F\left(u_{n}^{\delta}\right)-y^{\delta}-F^{\prime}\left(u_{n}^{\delta}\right) e_{n}\right\| \leq \frac{\mu_{n}}{\nu_{n}}\left\|y^{\delta}-F\left(u_{n}^{\delta}\right)\right\|
$$

In this case, Hanke proposes to choose $h_{n}$ based on the following discrepancy principle

$$
p_{n}^{\delta}\left(h_{n}\right):=\left\|F\left(u_{n}^{\delta}\right)-y^{\delta}-F^{\prime}\left(u_{n}^{\delta}\right)\left(u_{n}^{\delta}-u_{n+1}^{\delta}\right)\right\|=\mu_{n}\left\|y^{\delta}-F\left(u_{n}^{\delta}\right)\right\|,
$$

see [6, Section 2]. For later use we note the identity

$$
p_{n}^{\delta}(h)=\left\|\left(I+h K\left(u_{n}^{\delta}\right)\right)^{-1}\left(F\left(u_{n}^{\delta}\right)-y^{\delta}\right)\right\| .
$$

Moreover, explicit and implicit Runge-Kutta methods for linear problems were proposed and analyzed in [19]. It has been shown that for variable step sizes, Runge-Kutta approximations converge with optimal rate. For nonlinear problems, the convergence of linearly implicit Runge-Kutta methods was proved in [1]. The analysis, however, required severe restrictions on the time steps, see [1, Sect. 3] for details. 


\section{NeW REGULARIZATION METHOD}

Instead of solving (4) by a standard time integration scheme such as explicit, implicit or linearly implicit Runge-Kutta methods, we suggest to use a variant of exponential Runge-Kutta methods. Such methods have been studied recently in the context of time-dependent partial differential equations in $[10,11]$. The simplest scheme is the following variant of the exponential Euler method

$$
u_{n+1}^{\delta}=u_{n}^{\delta}-h_{n} \varphi\left(-h_{n} J\left(u_{n}^{\delta}\right)\right) F^{\prime}\left(u_{n}^{\delta}\right)^{*}\left(F\left(u_{n}^{\delta}\right)-y^{\delta}\right), \quad t_{n+1}=t_{n}+h_{n}, \quad n=0,1,2, \ldots
$$

where $\varphi$ denotes the entire function

$$
\varphi(z)=\frac{\mathrm{e}^{z}-1}{z}=1+\frac{z}{2}+\frac{z^{2}}{6}+\ldots
$$

Instead of the exact Jacobian again the operator $J\left(u_{n}^{\delta}\right)$ is used as argument of the matrix function.

In case of a linear problem where $F(v)=A v$, an exponential integrator solves the corresponding Showalter equation

$$
\frac{\mathrm{d}}{\mathrm{d} t} v^{\delta}(t)=-A^{*}\left(A v^{\delta}-y^{\delta}\right), \quad v^{\delta}(0)=v_{0}
$$

exactly for arbitrarily large time steps. This motivates the use of exponential integrators for nonlinear problems. Note that for linear problems this approach has been successfully used in [13].

Nonlinear problems are frequently treated with Newton's method. Linearizing (2) at the current approximation $u_{n}^{\delta}$ results in the linearized problem

$$
F^{\prime}\left(u_{n}^{\delta}\right) \Delta u_{n}^{\delta}=y^{\delta}-F\left(u_{n}^{\delta}\right)=: \Delta F_{n}
$$

with the update

$$
u_{n+1}^{\delta}=u_{n}^{\delta}+\Delta u_{n}^{\delta} .
$$

Solving the Showalter differential equation for the linear problem (13) with initial value $v^{\delta}(0)=0$ gives

$$
\Delta u_{n}^{\delta}=h_{n} \varphi\left(-h_{n} J\left(u_{n}^{\delta}\right)\right) F^{\prime}\left(u_{n}^{\delta}\right)^{*} \Delta F_{n} .
$$

This yields the same new approximation $u_{n+1}^{\delta}$ as the exponential Euler method applied to the nonlinear Showalter differential equation (4). Therefore, the exponential Euler method can be interpreted as Newton's method with asymptotic (Showalter) regularization for solving the linearized problems.

\section{Convergence properties: the continuous Case}

In [22], Tautenhahn analyzed the properties of the exact solution of (4). As these results serve as a guideline and framework for our numerical analysis, we will first review the assumptions and main results of this paper. The first assumption turns out to be important for controlling the linearization error of a Newton step.

Assumption 1. There exists $\eta<1$ such that

$$
\left\|F(\widetilde{u})-F(u)-F^{\prime}(u)(\widetilde{u}-u)\right\| \leq \eta\|F(\widetilde{u})-F(u)\|,
$$

for all $u, \widetilde{u}$ in a ball $B_{r}\left(u_{0}\right)$ of radius $r>0$ around the center $u_{0}$.

Under our general smoothness requirements on $F$ and the above assumption, the asymptotic regularization approach converges for $\delta \rightarrow 0$. More precisely, the following result given in [22, Thm. 4] holds. 
Theorem 3.1. Consider problem (2) with noisy initial data (3). Let Assumption 1 and $\left\|F\left(u_{0}\right)-y^{\delta}\right\|>\tau \delta>0$ be satisfied, and let $u^{\delta}(T)$ be the solution of (4), where the stopping time $T=T(\delta)$ is chosen in accordance with the discrepancy principle $(5)$ with $\tau>(1+\eta) /(1-\eta)$. If $(1)$ is solvable in $B_{r}\left(u_{0}\right)$, then $u^{\delta}(T)$ converges to a solution of (1) for $\delta \rightarrow 0$.

In [22], it is further shown that the discrepancy principle implies a finite stopping time $T$.

For a proof of optimal convergence rates, two additional assumptions are required. The first one concerns the source representation. Recall that $u_{+}$denotes the solution of (1) with minimal distance to $u_{0}$.

Assumption 2. There exists $w \in X$ and constants $\gamma \in(0,1 / 2]$ and $\rho \geq 0$ such that

$$
e_{0}=u_{0}-u_{+}=J\left(u_{+}\right)^{\gamma} w, \quad\|w\| \leq \rho .
$$

The last assumption finally allows to represent the Fréchet derivative $F^{\prime}(u)$ for $u$ close to $u_{0}$ by $F^{\prime}\left(u_{+}\right)$.

Assumption 3. For all $u \in B_{r}\left(u_{0}\right)$ there exists a linear bounded operator $R_{u}: Y \rightarrow Y$ and a constant $C_{+} \geq 0$ such that

(1) $F^{\prime}(u)=R_{u} F^{\prime}\left(u_{+}\right)$

(2) $\left\|R_{u}-I\right\| \leq C_{+}\left\|u-u_{+}\right\|$.

In the linear case this assumption is always satisfied with $R_{u}=I$. Without loss of generality, we can further assume that

$$
\left\|F^{\prime}(u)\right\| \leq 1, \quad u \in B_{r}\left(u_{0}\right)
$$

The following theorem states that, under the above assumptions, the convergence rate is optimal. This result is again taken from Tautenhahn, see [22, Thm.6].

Theorem 3.2. Let the assumptions of Theorem 3.1 hold. If in addition Assumptions 2-3 and (17) are satisfied and if $\tau>(2-\eta) /(1-\eta)$, then there exists a constant $c_{*}=c_{*}\left(\gamma, \tau, \eta, C_{+}\right)$such that

$$
\left\|u^{\delta}(T)-u_{+}\right\| \leq c_{*} \rho^{1 /(2 \gamma+1)} \delta^{2 \gamma /(2 \gamma+1)}
$$

for $\rho$ sufficiently small.

Since $\gamma \in(0,1 / 2]$, the theorem gives $\sqrt{\delta}$ as best possible rate for the convergence $u^{\delta}(T) \rightarrow u_{+}$.

\section{Convergence properties: the discrete CASe}

In this section, we present our convergence results for the new regularization method based on exponential integrators. The proofs are quite involved and will be published elsewhere, see [8].

Since our integrator can cope with large eigenvalues, we are in particular interested in variable step sizes that can grow unboundedly for $t \rightarrow \infty$. In [8] we proved that one can select the step sizes $h_{n}$ such that the errors are monotonically decreasing. For this, let $0<\mu \leq \mu_{n}$ be a sequence of parameters, and define

$$
\psi(z)=\varphi(z)(1-z)
$$

Then the step size $h_{n}$ for our method (10) is chosen based on the following discrepancy principle

$$
\left\|\psi\left(-h_{n} K\left(u_{n}^{\delta}\right)\right) \Delta F_{n}-F^{\prime}\left(u_{n}^{\delta}\right) \Delta u_{n}^{\delta}\right\|=\mu_{n}\left\|\Delta F_{n}\right\| .
$$

Equivalently, we can write it as

$$
\left\|\varphi\left(-h_{n} K\left(u_{n}^{\delta}\right)\right) \Delta F_{n}\right\|=\mu_{n}\left\|\Delta F_{n}\right\|
$$


Remark. For the choice $\varphi(x)=(1-x)^{-1}$, method (10) together with (18)-(19) corresponds to the LevenbergMarquardt method with step size selection (8) and (9) proposed in [6]. In this case, we have $\psi(x) \equiv 1$.

To minimize the computational effort the parameters $\mu_{n}$ have to be carefully chosen. Our numerical experiments (see Section 6) showed that the parameter selection by Rieder [16] yields good results.

We are able to show convergence of our discrete regularization method under similar assumptions as Tautenhahn does for the asymptotic regularization.

Theorem 4.1. Let $(1+\eta+\tau(0.3+\eta)) / \tau<\mu<1$. Assume that (17), Assumption 1 , and (3) hold. If the step sizes $h_{n}$ are chosen according to (18), then the exponential Euler iteration stopped by the discrepancy principle (5) terminates after $n_{*}=n_{*}(\delta)<\infty$ iterations and the corresponding approximations $u_{n_{*}}$ converge to a solution of (1) as $\delta \rightarrow 0$.

To the best of our knowledge it is not possible to prove optimal convergence rates in the discrete case under the same assumptions as in the continous case. Nevertheless, under slight restrictions to the time step sizes $h_{n}$, we are able to prove the optimality of our method.

Theorem 4.2. Let Assumptions 1 to 3 and (3) hold and assume that the step sizes $h_{j}$ defined by (18) are monotonically increasing and satisfy

$$
h_{j} \leq c_{h}\left(1+t_{j}\right)^{\gamma-\epsilon}, \quad \epsilon>0 .
$$

Then, for $\rho$ sufficiently small, there exists a constant $C>0$ such that the error of the exponential Euler iteration stopped by the discrepancy principle (5) with $\tau>(2-\eta) /(1-\eta)$ satisfies

$$
\left\|e_{n_{*}}\right\| \leq C \rho^{1 /(2 \gamma+1)} \delta^{2 \gamma /(2 \gamma+1)} .
$$

The proofs of these two theorems are given in [8].

\section{Implementation}

In numerical computations we have to deal with a discrete version of (4). However, for readability reasons we use the same notation for the discrete problem as above but fix the index $n$ specifying the time step.

The main computational effort per time step consists in the approximation of the products of a matrixfunction with a vector. This is necessary for (10) and for the step size selection (19). Since the matrices are symmetric, these products are approximated by the symmetric Lanczos process. By writing (10) in the equivalent form

$$
u_{n+1}^{\delta}=u_{n}^{\delta}-h_{n} F^{\prime}\left(u_{n}^{\delta}\right)^{*} \varphi\left(-h_{n} K\left(u_{n}^{\delta}\right)\right) \Delta F_{n},
$$

one can use the same Krylov subspace for both approximations. To be more precise, let $V_{m}=\left[v_{1} \ldots, v_{m}\right]$ be an orthonormal basis of the $m$ th Krylov subspace

$$
\mathcal{K}_{m}\left(K\left(u_{n}^{\delta}\right), \Delta F_{n}\right)=\operatorname{span}\left\{\Delta F_{n}, K\left(u_{n}^{\delta}\right) \Delta F_{n}, \ldots, K\left(u_{n}^{\delta}\right)^{m-1} \Delta F_{n}\right\}
$$

Then $H_{m}=V_{m}^{*} K\left(u_{n}^{\delta}\right) V_{m} \in \mathbb{R}^{m, m}$ is a tridiagonal matrix and the Krylov relation reads

$$
K\left(u_{n}^{\delta}\right) V_{m}=V_{m} H_{m}+\beta_{m+1} v_{m+1} e_{m}^{T}, \quad m=1,2, \ldots
$$

where $\beta_{m+1}$ denotes the $(m+1, m)$ element of $H_{m+1}$ and $e_{i}$ is the $i$ th unit vector in $\mathbb{R}^{m}$. This enables to approximate

$$
\varphi\left(-h_{n} K\left(u_{n}^{\delta}\right)\right) \Delta F_{n} \approx V_{m} \varphi\left(-h_{n} H_{m}\right) e_{1}\left\|\Delta F_{n}\right\|
$$

For a convergence analysis of Krylov approximations to matrix functions we refer to [3,9]. To avoid loss of orthogonality within the Lanczos vectors, we applied reorthogonalization [2]. 
We have to terminate this process as soon as our approximation is within a predefined distance to the sought-after vector. We approximate the error $\epsilon_{m}$ by

$$
\left\|\epsilon_{m}\right\| \lesssim \frac{\delta_{m}}{1-\delta_{m}}\left\|w_{m}\right\|
$$

(cf. [23]) where $w_{m}:=\varphi\left(-h H_{m}\right) e_{1}$ and

$$
\delta_{m}=\frac{\left\|w_{m}-w_{m-k}\right\|}{\left\|w_{m}\right\|}, \quad k \geq 1
$$

is an approximation of the relative error in step $m$. Our experiments show that this is a reliable and efficient estimate for this problem. Actually, since we are not interested in a high accuracy solution of the initial value problem (4) but in the solution of the ill-posed problem (2) we can use quite large tolerances. We achieved good results in the model problems from Section 6 with tolerances in the order of the norm of the noise $\delta$.

Due to this implementation we do neither need a decomposition of $K\left(u_{n}^{\delta}\right)$ nor have to solve linear systems. The symmetric Lanczos process just needs matrix-vector products with the matrices $F^{\prime}\left(u_{n}^{\delta}\right)$ and $F^{\prime}\left(u_{n}^{\delta}\right)^{*}$. The matrices $K\left(u_{n}^{\delta}\right), F^{\prime}\left(u_{n}^{\delta}\right)$ and $F^{\prime}\left(u_{n}^{\delta}\right)^{*}$ are not required explicitly, i.e., a matrix free implementation is possible.

\section{NumericAl EXAMPLES}

In the first example, we identify a coefficient of an elliptic PDE from the solution, the second example is taken from hydrology.

\subsection{Parameteridentification in elliptic PDEs}

The first example is taken from [16]. We aim at identifying the non-negative coefficient $u=u(\xi, \eta)$ in the two-dimensional elliptic problem

$$
\begin{aligned}
-\Delta y+u y=f & & \text { in } \Omega \\
y=g & & \text { on } \partial \Omega
\end{aligned}
$$

from the knowledge of the solution $y=y(\xi, \eta)$ in $\Omega=(0,1)^{2}$. The source function $f$ and the boundary data $g$ are chosen such that

$$
y(\xi, \eta)=16 \xi(\xi-1) \eta(1-\eta)+1
$$

is the solution of (25) with coefficient

$$
u_{+}(\xi, \eta)=1.5 \sin (2 \pi \xi) \sin (3 \pi \eta)+3\left((\xi-0.5)^{2}+(\eta-0.5)^{2}\right)+2 .
$$

The latter has to be identified. This problem fits into our abstract framework with $X=Y=L^{2}(\Omega)$, see also [16].

For its numerical solution, the problem is discretized in space by standard finite differences on a regular, uniform grid with $N$ internal points in each direction. To obtain a matrix-vector representation of the discrete problem, we reorder the grid points lexicographically. This results in the finite dimensional representation

$$
F(u):=(A+\operatorname{diag}(u))^{-1} f=y^{\delta},
$$

where the square matrix $-A$ is the standard five point discretization of the Laplacian. For the numerical solution of (26), we endow the underlying space $\mathbb{R}^{N \times N}$ with the Euclidian norm

$$
\|z\|_{N}=\frac{1}{N} \sqrt{\sum_{j=1}^{N^{2}} z_{j}^{2}}
$$



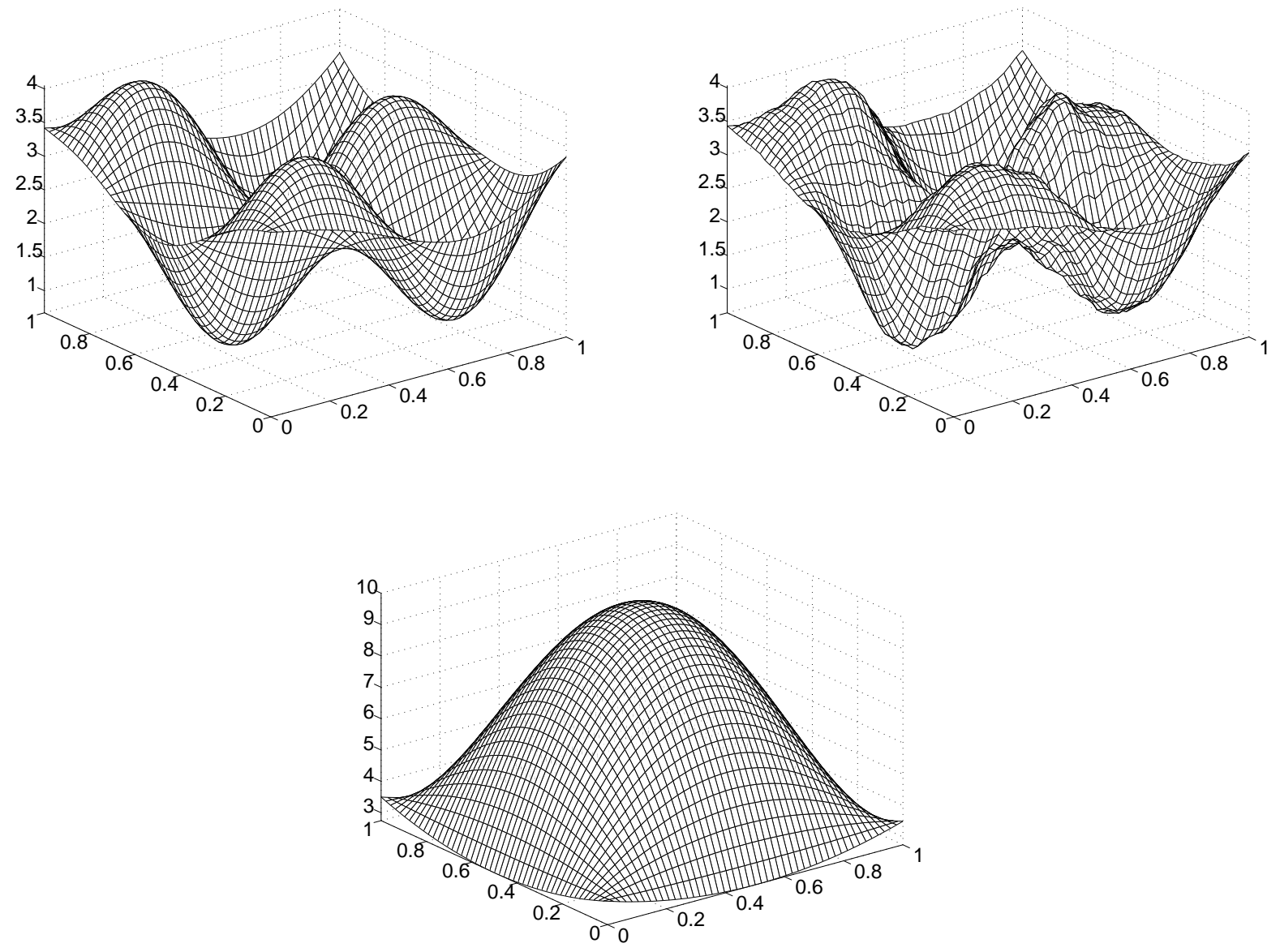

FiguRE 1. The coefficient $u_{+}(\xi, \eta)$ (top left), its reconstruction $u_{n_{*}}^{\delta}(\xi, \eta)$ (top right), and the initial value $u_{0}(\xi, \eta)$ (bottom) of the Showalter equation

This is an approximation to the $L^{2}(\Omega)$ norm of the continuous problem. The nonlinear function $F$ is differentiable with Fréchet derivative $F^{\prime}(u)$ given by

$$
F^{\prime}(u) w=-(A+\operatorname{diag}(u))^{-1} \operatorname{diag}(w)(A+\operatorname{diag}(u))^{-1} f, \quad w \in \mathbb{R}^{N^{2}} .
$$

The perturbed right-hand side $y^{\delta}$ of $(26)$ is defined as

$$
y^{\delta}=y+\delta z /\|z\|_{N}
$$

where the entries of $z$ are uniformly distributed random variables in $[-1,1]$. As initial value for the Showalter equation, we choose the function

$$
u_{0}(\xi, \eta)=3\left((\xi-0.5)^{2}+(\eta-0.5)^{2}\right)+2+128 \xi(\xi-1) \eta(1-\eta) .
$$

Since $u_{0}-u_{+}$is smooth and satisfies homogeneous Dirichlet boundary conditions, we obtain $\gamma=1 / 2$ in the source condition (16). 


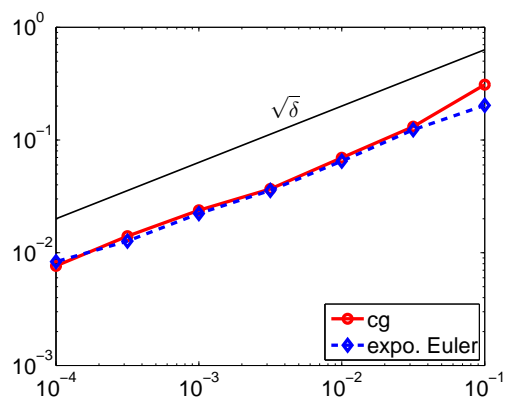

FIGURE 2. Reconstruction error as function of the perturbation parameter $\delta$ (exponential Euler method vs. cg-REGINN)
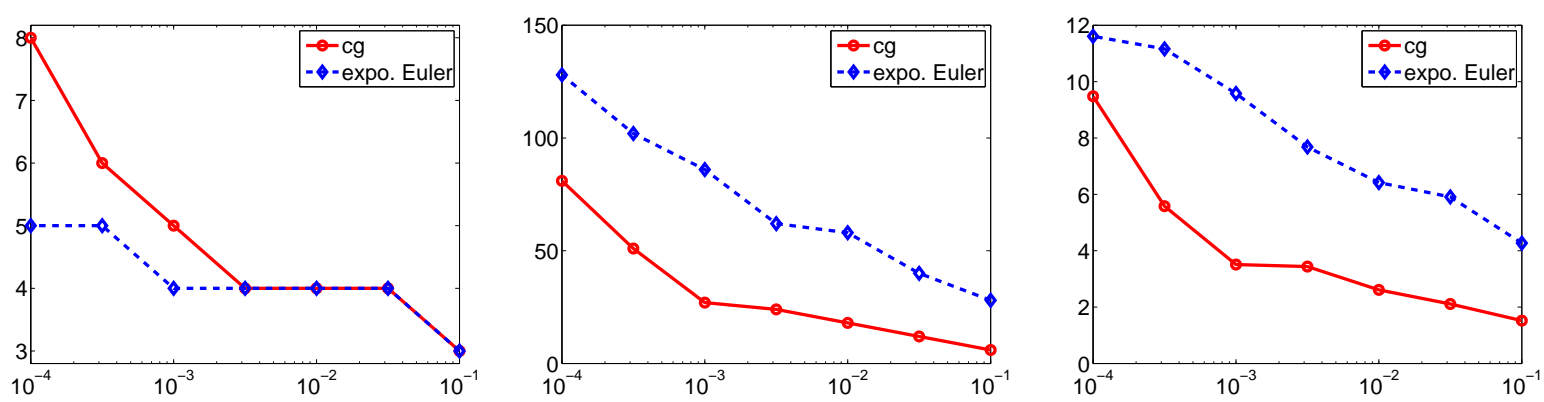

FiguRE 3. Number of outer iterations (left), overall matrix-vector-multiplications (middle) and cpu-time (right) as function of the perturbation parameter $\delta$ (exponential Euler method vs. cg-REGINN)

Using $N=50, \delta=10^{-2.5}, \tau=1.5$ and constant $\mu_{n}=0.95$, the exponential Euler method gives a regularization with a relative error of $\left\|u_{n_{*}}^{\delta}-u_{+}\right\|_{N} /\left\|u_{+}\right\|_{N} \approx 5 \%$, see Figure 1 .

Figure 2 shows the regularization property of our scheme compared to the regularization based on inexact Newton iteration with conjugate gradient method as inner iteration (cg-REGINN [18]). The figure demonstrates that both regularization schemes reflect the expected error decay $\sqrt{\delta}$ as $\delta \rightarrow 0$ from Theorem 4.2. Figure 3 reveals that both schemes require approximately the same work for small $\delta$.

\subsection{Inverse problem in groundwater hydrology}

The second example is taken from [6]. We consider the boundary value problem

$$
\begin{aligned}
-\operatorname{div}(u \operatorname{grad} y) & =f & & \text { in } \Omega \\
y & =g & & \text { on } \partial \Omega
\end{aligned}
$$

in $\Omega=[0,6]^{2}$ with mixed Dirichlet-Neumann boundary data

$$
y(\xi, 0)=100, \quad y_{\xi}(6, \eta)=0, \quad\left(u y_{\xi}\right)(0, \eta)=-500, \quad y_{\eta}(\xi, 6)=0
$$

and right-hand side

$$
f(\xi, \eta)= \begin{cases}0 & 0<\eta<4 \\ 137 & 4<\eta<5 \\ 274 & 5<\eta<6 .\end{cases}
$$


Again we are interested to identify the coefficient. This time, it is a piecewise constant function $u_{+}$displayed in Figure 4. This problem serves as a model problem for steady states in groundwater filtration. The searched coefficient $u_{+}$plays the role of the diffusivity of the sediment. For details, we refer to [6].

Since $u_{+}$is discontinuous, our assumptions are not satisfied and the theoretical results from Section 3 do not apply to this example. For details, see [6]. However, our numerical experiments show the usefulness of our regularization scheme for a larger class of problems.

We implimented the inverse problem from (27) using finite elements. Figure 4 shows the exact solution and a reconstruction of $u_{+}$with $\delta=0.001$ on a triangulation with 288 triangles and 169 grid pionts. The relative
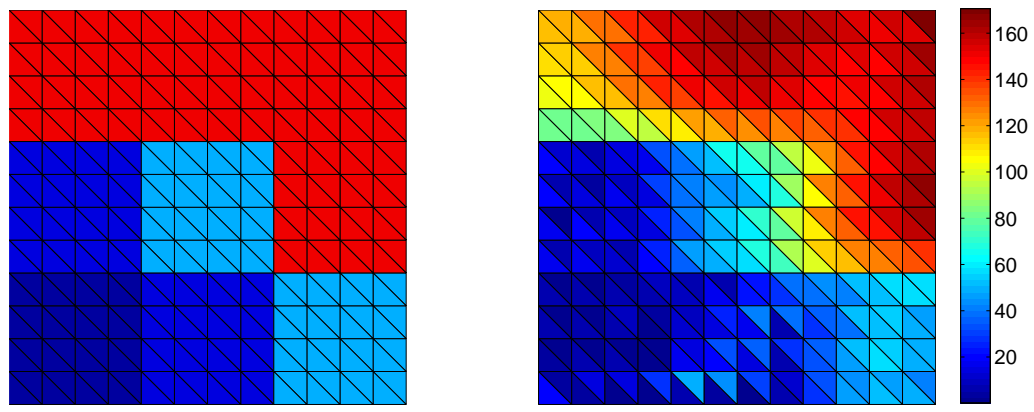

FigurE 4. The diffusivity $u_{+}$(left) and its reconstruction $u_{n_{*}}^{\delta}$ (right)

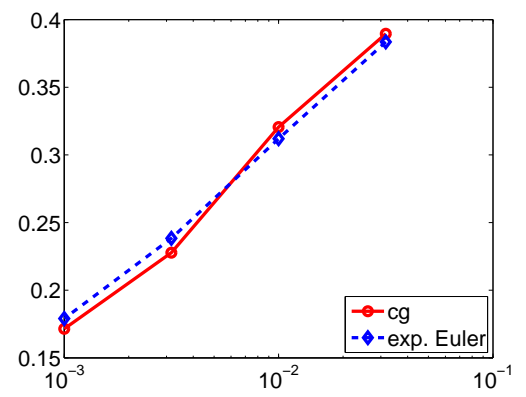

FIGURE 5. Reconstruction error as function of the perturbation parameter $\delta$ (exponential Euler method vs. cg-REGINN)
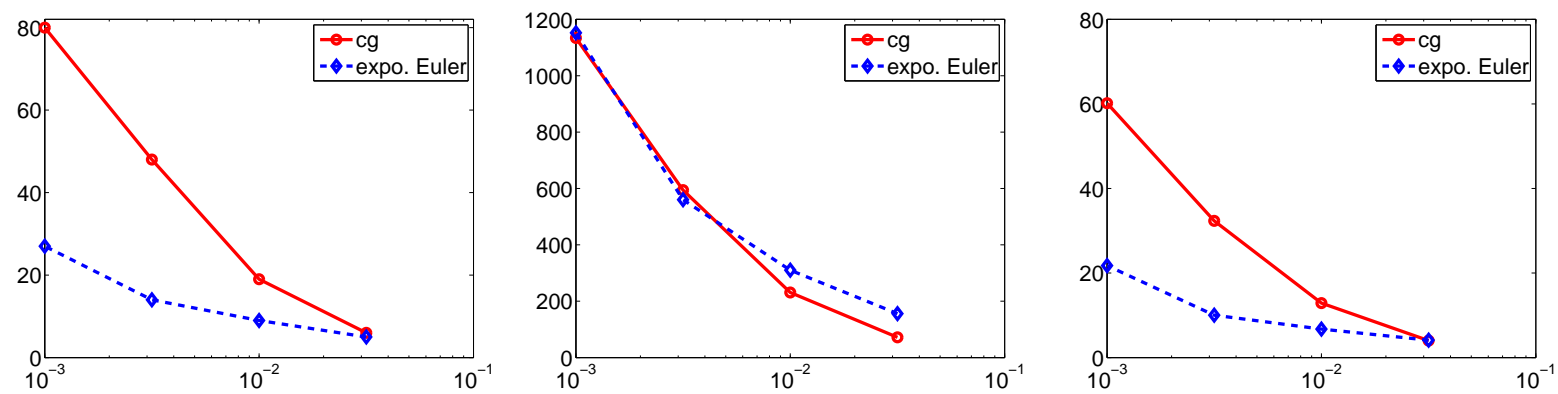

FIgURE 6. Number of outer iterations (left), overall matrix-vector-multiplications (middle) and cpu-time (right) as function of the perturbation parameter $\delta$ (exponential Euler method vs. cg-REGINN) 
error is 18\%. The computations of Figure 5 and 6 are done on a finer grid with 1152 triangles and 625 grid points.

Again, as in the first example both regularization schemes (exponential Euler method and cg-REGINN) show the same error decay, see Figure 5. Furthermore, Figure 6 shows that both methods require approximately the same number of matrix-vector products though the exponential Euler scheme needs significantly less outer iterations than cg-REGINN. Since every nonlinear step itself is expensive, this indicates that our scheme is much more efficient for this problem.

\section{Concluding Remarks}

In this paper we have considered exponential integrators for the regularization of nonlinear ill-posed problems. We have concentrated on a variant of the exponential Euler method, but we strongly believe that higher order exponential integrators will have favorable regularization properties as well. Our convergence proofs given in [8] only cover the case of the exponential Euler method. An extension of these proofs to higher order methods seems to be possible even though quite technical.

Our numerical experiments clearly indicate that both the exponential Euler method and the cg-REGINN scheme show a similar error decay as the perturbation tends to zero. For small perturbations, the number of outer iterations, however, is significantly smaller for the exponential Euler scheme. In situations when this nonlinear step is expensive we obtain computational efficiency of the exponential Euler method. This fact can be observed in the second example from groundwater hydrology.

\section{ACKNOWLEDGEMENT}

The authors wish to thank Martin Hanke for helpful discussions and for providing a MATLAB code for the numerical example in Section 6.2.

\section{REFERENCES}

[1] C. Böckmann and P. Pornsawad. Iterative Runge-Kutta-type methods for nonlinear ill-posed problems. Inverse Problems, $24(2), 2008$.

[2] J. Daniel, W.B. Gragg, L. Kaufman, and G.W. Stewart. Reorthogonalization and stable algorithms for updating the GramSchmidt QR factorization. Math. Comp, 30:772-795, 1976.

[3] V. L. Druskin and L. A. Knizhnerman. Krylov subspace approximations of eigenpairs and matrix functions in exact and computer arithmetic. Numer. Lin. Alg. Appl., 2:205-217, 1995.

[4] H. W. Engl, K. Kunisch, and A. Neubauer. Convergence rates for Tikhonov regularization of nonlinear ill-posed problems. Inverse Problems, 5:523-540, 1989.

[5] B. Hackl. Geometry Variations, Level Set and Phase-field Methods for Perimeter Regularized Geometric Inverse Problems. $\mathrm{PhD}$ thesis, Johannes Keppler Universität Linz, 2006.

[6] M. Hanke. A regularizing Levenberg-Marquardt scheme, with applications to inverse groundwater filtration problems. Inverse Problems, 13:79-95, 1997.

[7] M. Hanke, A. Neubauer, and O. Scherzer. A convergence analysis of the Landweber iteration for nonlinear ill-posed problems. Numerische Mathematik, 72(1):21-37, 1995.

[8] M. Hochbruck, M. Hönig, and A. Ostermann. A convergence analysis of the exponential Euler iteration for nonlinear ill-posed problems. Technical report, University of Düsseldorf, Germany, 2008.

[9] M. Hochbruck and C. Lubich. On Krylov subspace approximations to the matrix exponential operator. SIAM J. Numer. Anal., 34(5):1911-1925, 1997.

[10] M. Hochbruck and A. Ostermann. Explicit exponential Runge-Kutta methods for semilinear parabolic problems. SIAM J. Numer. Anal., 43(3):1069-1090, 2005.

[11] M. Hochbruck, A. Ostermann, and J. Schweitzer. Exponential Rosenbrock-type methods. SIAM J. Numer. Anal., to appear.

[12] T. Hohage and S. Langer. Convergence analysis of an inexact iteratively regularized Gauss-Newton method under general source conditions. Journal of Inverse and Ill-Posed Problems, 15:19-35, 2007.

[13] M. Hönig. Asymptotische Regularisierung schlecht gestellter Probleme mittels steifer Integratoren. Diplomarbeit, Universität Karlsruhe, 2004

[14] B. Kaltenbacher, A. Neubauer, and O. Scherzer. Iterative Regularization Methods for Nonlinear Ill-Posed Problems. de Gruyter, Berlin, New York, 2008. 
[15] A. Neubauer. Tikhonov regularization for non-linear ill-posed problems: optimal convergence rates and finite-dimensional approximation. Inverse Problems, 5:541-557, 1989.

[16] A. Rieder. On the regularization of nonlinear ill-posed problems via inexact Newton iterations. Inverse Problems, 15(1):309$327,1999$.

[17] A. Rieder. On convergence rates of inexact Newton regularizations. Numerische Mathematik, 88(2):347-365, 2001.

[18] A. Rieder. Inexact Newton regularization using conjugate gradients as inner iteration. SIAM J. Numer. Anal., 43:604-622, 2005.

[19] A. Rieder. Runge-Kutta integrators yield optimal regularization schemes. Inverse Problems, 21(2):453-471, 2005.

[20] T. I. Seidman and C. R. Vogel. Well-posedness and convergence of some regularization methods for nonlinear ill-posed problems. Inverse Problems, 5:227-238, 1989.

[21] D. Showalter. Representation and computation of the pseudoinverse. Proceedings of the American Mathematical Society, 18:584-586, 1967.

[22] U. Tautenhahn. On the asymptotical regularization of nonlinear ill-posed problems. Inverse Problems, 10(6):1405-1418, 1994.

[23] J. van den Eshof and M. Hochbruck. Preconditioning Lanczos approximations to the matrix exponential. SIAM J. Sci. Comp., $27: 1438-1457,2006$. 